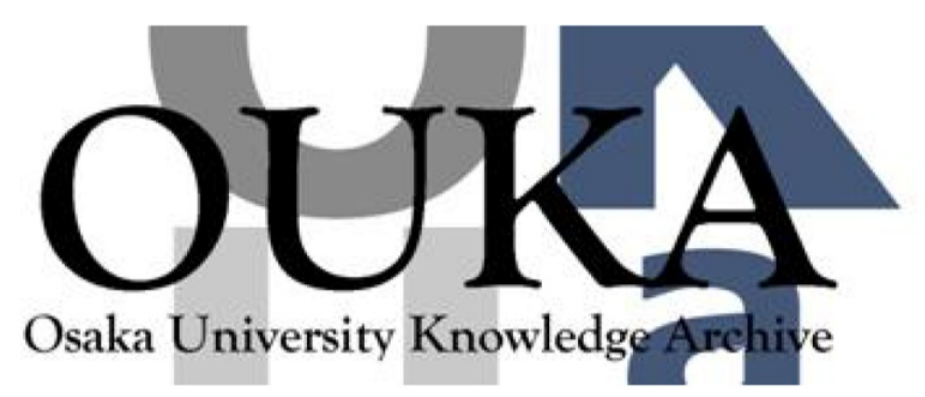

\begin{tabular}{|c|l|}
\hline Title & $\begin{array}{l}\text { Analysis of defect mode switching response in } \\
\text { one-dimensional photonic crystal with a nematic } \\
\text { liquid crystal defect layer }\end{array}$ \\
\hline Author(s) & $\begin{array}{l}\text { Ozaki, Ryotaro; Moritake, Hiroshi; Yoshino, } \\
\text { Katsumi et al. }\end{array}$ \\
\hline Citation & $\begin{array}{l}\text { Journal of Applied Physics. 101(3) p. 033503- } \\
\text { p. } 033503\end{array}$ \\
\hline Issue Date & $2007-02-01$ \\
\hline oaire:version & VoR \\
\hline URL & https://hdl.handle. net/11094/75846 \\
\hline rights & \\
\hline Note & \\
\hline
\end{tabular}

Osaka University Knowledge Archive : OUKA

https://ir. Library. osaka-u. ac. jp/

Osaka University 


\section{Analysis of defect mode switching response in one-dimensional photonic crystal with a nematic liquid crystal defect layer}

Cite as: J. Appl. Phys. 101, 033503 (2007); https://doi.org/10.1063/1.2432877

Submitted: 29 June 2006 . Accepted: 22 November 2006 . Published Online: 01 February 2007

Ryotaro Ozaki, Hiroshi Moritake, Katsumi Yoshino, and Masanori Ozaki

\section{ARTICLES YOU MAY BE INTERESTED IN}

Electrically color-tunable defect mode lasing in one-dimensional photonic-band-gap system containing liquid crystal

Applied Physics Letters 82, 3593 (2003); https://doi.org/10.1063/1.1577829

High $Q$ defect mode and laser action in one-dimensional hybrid photonic crystal containing cholesteric liquid crystal

Applied Physics Letters 89, 101109 (2006); https://doi.org/10.1063/1.2347114

Electrically tunable lasing based on defect mode in one-dimensional photonic crystal with conducting polymer and liquid crystal defect layer

Applied Physics Letters 84, 1844 (2004); https://doi.org/10.1063/1.1686891

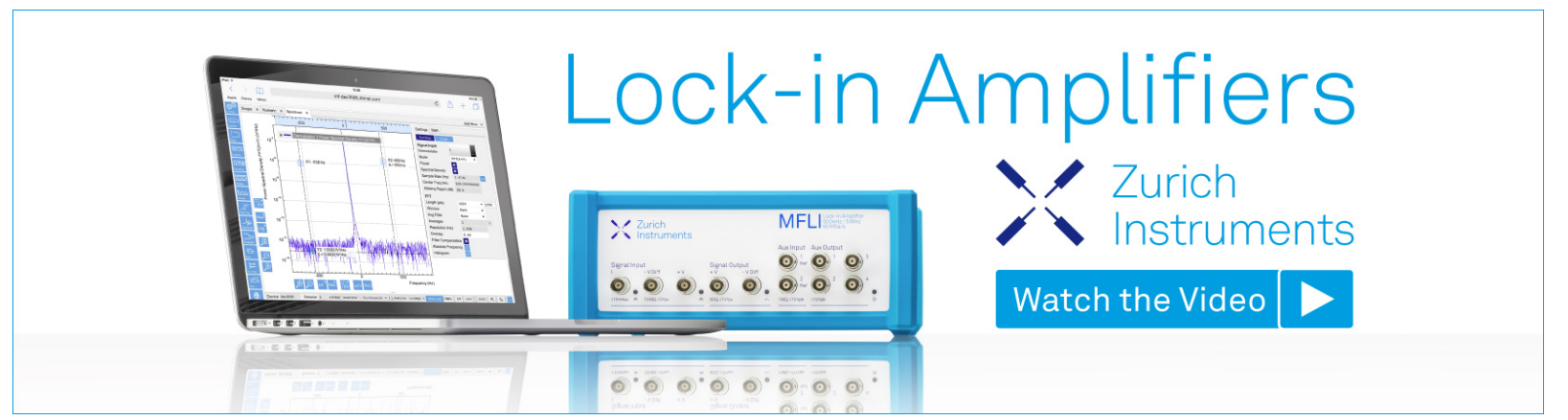




\title{
Analysis of defect mode switching response in one-dimensional photonic crystal with a nematic liquid crystal defect layer
}

\author{
Ryotaro Ozaki ${ }^{\text {a) }}$ and Hiroshi Moritake \\ Department of Electrical and Electronic Engineering, National Defense Academy, 1-10-20 Hashirimizu, \\ Yokosuka, Kanagawa 239-8686, Japan \\ Katsumi Yoshino \\ Research Project Promotion Institute, Shimane University, 2 Hokuryo-cho, Matsue, \\ Shimane 690-0816, Japan \\ Masanori Ozaki \\ Division of Electrical, Electronic, and Information Engineering, Graduate School of Engineering, \\ Osaka University, 2-1 Yamada-Oka, Suita, Osaka 565-0871, Japan
}

(Received 29 June 2006; accepted 22 November 2006; published online 1 February 2007)

\begin{abstract}
We analyze the dynamic response and optical properties of a high-speed defect mode switching that is based on a tunable defect mode in a one-dimensional photonic crystal with a nematic liquid crystal defect layer. The electro-optic responses of the defect mode switching are calculated considering the director distributions in the defect layer, which are determined using continuum theory. The calculated defect mode switching exhibits a response on the order of microseconds in spite of the use of the reorientation of nematic liquid crystal molecules. From the calculation, it is found that the fast response is realized using a narrow peak shift and a fast part of the molecular reorientation. Furthermore, the dependences of the switching response are clarified on several physical parameters. (C) 2007 American Institute of Physics. [DOI: 10.1063/1.2432877]
\end{abstract}

\section{INTRODUCTION}

Photonic crystals (PCs) having a three-dimensional ordered structure with a periodicity equivalent to the optical wavelength are among the most interesting optical materials, because they have a photonic band gap (PBG) in which the existence of photons is forbidden in a certain energy range. ${ }^{1,2}$ Using this feature, considerable advancements in optical devices, such as low-threshold lasers, microwaveguides, and optical circuits, are expected. ${ }^{1-5}$

Tunable PCs having variable PBGs by controlling optical periodicities are also expected for functional applications, for example, tunable lasers and tunable optical waveguides. In particular, liquid crystals (LCs) in PCs have attracted much attention and have been studied for the realization of tunable PCs. ${ }^{6-10}$ In these studies, LC is used as a periodic structure for controlling the PBG, because LC has a large optical anisotropy and is sensitive to external fields such as temperature and electric and magnetic fields. Controlling the external field to the LC produces a change in the period length of the PC. In the cases of opals or inverse opals filling with nematic LC (NLC), tunable PBGs have been demonstrated by applying an electric field or controlling temperature.

On the other hand, using NLC as a defect in PC has also been researched. ${ }^{11-13}$ We have fabricated a one-dimensional (1D) PC containing a NLC defect layer and have demonstrated the electrical tuning of a defect mode wavelength. ${ }^{11}$ Furthermore, the modulation of defect mode lasing by applying a low voltage has also been demonstrated in the 1D PC

${ }^{\text {a)} E l e c t r o n i c ~ m a i l: ~ o z a k i @ n d a . a c . j p ~}$ with a dye-doped NLC defect. ${ }^{12}$ The 1D PC with the NLC defect layer is used not only for the tunable laser but also for the defect mode switching. ${ }^{13}$ The switching is based on a peak shift of a defect mode, and the response is very fast. Although it is well known that the response time of NLC is on the order of milliseconds, defect mode switching having a response on the order of tens of microseconds has been demonstrated experimentally. However, defect mode switching has not yet been analyzed theoretically. In this paper, we analyze the electro-optic response of the defect mode switching in the 1D PC with the NLC defect layer, considering the molecular director distributions in the defect layer.

\section{ANALYSIS METHOD}

A schematic view of a 1D PC with a NLC defect layer is shown in Fig. 1. The 1D PC with the NLC defect is composed of dielectric multilayers and the NLC defect layer located at the center of the dielectric multilayers. The dielectric multilayer as a 1D PC consists of an alternating stack of $\mathrm{TiO}_{2}$ and $\mathrm{SiO}_{2}$ layers, and the periodicity of one side of the multilayer involves five pairs. The initial orientation of the NLC molecules is set along the $z$ axis. The molecular directions in the defect layer are controlled by applying voltage to the transparent electrodes shown in Fig. 1. Since NLC has a large anisotropy in its refractive index depending on molecular alignment, the redirection of the NLC molecules in the defect layer produces a change in the optical length. This indicates that the defect mode wavelength can be controlled by applying voltage to the NLC defect layer.

To perform an analysis that takes into account the NLC molecular reorientation induced by applying voltage, the NLC molecular alignment in the defect layer was calculated 


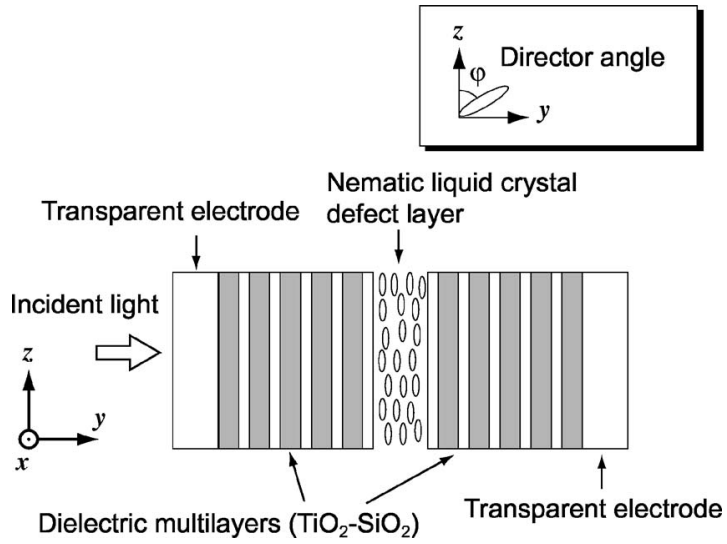

FIG. 1. Schematic view of one-dimensional photonic crystal (PC) with nematic liquid crystal (NLC) defect layer.

on the basis of the Franck continuum theory in which the NLC is regarded as an elastic continuum, and free energy, which includes both kinetic and electric energies, is taken into account. ${ }^{14}$ Using kinetic coefficients $K_{11}$ and $K_{33}$, director vector $\boldsymbol{n}$, and electric field $\boldsymbol{E}$, the free energy density $g$ of a splay transition in the LC cell is given by

$$
g=\frac{1}{2} K_{11}(\nabla \cdot \boldsymbol{n})^{2}+\frac{1}{2} K_{33}[\boldsymbol{n} \times(\nabla \times \boldsymbol{n})]^{2}-\frac{1}{2} \boldsymbol{\varepsilon}_{0} \boldsymbol{\varepsilon}_{a}(\boldsymbol{E} \cdot \boldsymbol{n})^{2},
$$

where $\varepsilon_{0}$ is the dielectric constant of vacuum and $\varepsilon_{a}$ is the dielectric anisotropy between the ordinary and extraordinary axes of the NLC molecules. Although a cyanobiphenyl-based mixture was used in our previous study, ${ }^{13}$ 4-cyano4'-pentylbiphenyl (5CB), which is a well-known typical $\mathrm{NLC}$, is used for analysis in this study. The dielectric anisotropy and elastic constants $K_{11}$ and $K_{33}$ of 5CB are 11.0, 6.37 , and $8.6 \mathrm{pN}$, respectively. Using the director angle $\varphi$ shown in Fig. 1, the free energy density $g$ is written as

$$
g=\frac{1}{2}\left[\left(K_{11} \cos ^{2} \varphi+K_{33} \sin ^{2} \varphi\right)\left(\frac{d \varphi}{d y}\right)^{2}-\varepsilon_{0} \varepsilon_{a} E \sin ^{2} \varphi\right] .
$$

The derivative of $\varphi$ is obtained on the basis of the EulerLagrange equation, and the director distribution for the static characteristic is determined by solving the differential equation of $\varphi$. On the other hand, the dynamic characteristic is determined by solving the following torque balance equation:

$$
\gamma \frac{\partial \varphi}{\partial t}=-\left[\frac{\partial g}{\partial \varphi}-\frac{d}{d y} \frac{\partial g}{\partial(d \varphi / d y)}\right],
$$

where $\gamma$ is the rotational viscosity of NLC. To calculate Eq. (3) numerically, it was approximated in time and space domains using a difference method, and we solved the differential equation with respect to $\varphi$. In the director angle calculation, the calculation resolution and rotational viscosity are set to $\Delta y=2 \mathrm{~nm}$ and $\gamma=0.06 \mathrm{~Pa}$ s, respectively.

The transmission characteristic of the 1D PC with the NLC defect layer was calculated by Berreman's $4 \times 4$ matrix method. ${ }^{15}$ This method is one of the numerical methods for

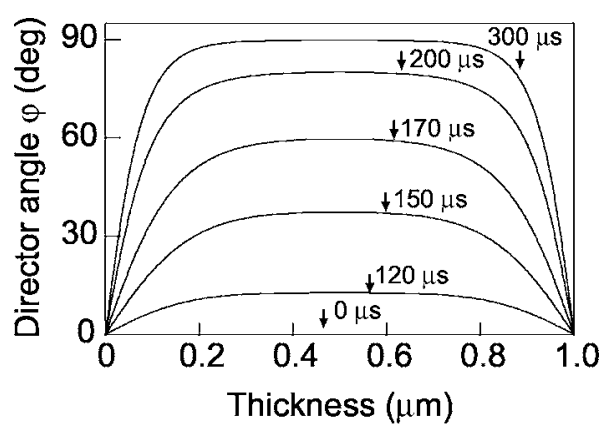

(a)

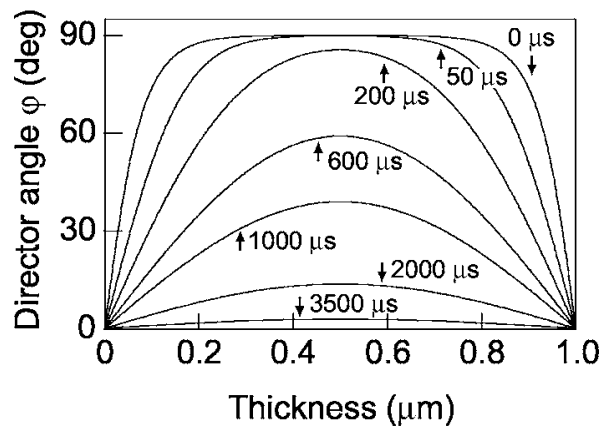

(b)

FIG. 2. Director distributions of the NLC molecules in the defect layer as a function of time. (a) Reorientations of the NLC molecules for an applied voltage of $15 \mathrm{~V}$. (b) Relaxations of the NLC molecules in the absence of applied electric field after removing the applied voltage.

solving Maxwell's equations and is appropriate for a propagation medium having a continuous change in refractive index. The light propagation along the $y$ axis with frequency $\omega$ is given by

$$
\frac{d \boldsymbol{\Psi}(y)}{d y}=\frac{i \omega}{c} \boldsymbol{D}(y) \boldsymbol{\Psi}(y),
$$

where $\boldsymbol{D}(y)$ is the propagation matrix and $\boldsymbol{\Psi}(y)$ $=\left(E_{x}, H_{z}, E_{z}, H_{x}\right)^{T}$. In this calculation, the refractive index distribution in the NLC defect layer was obtained from the director angle distribution determined from the above calculation. Therefore, the electro-optic response of the defect mode switching could be obtained from the calculation of transmission intensity considering the NLC molecular orientations. Here, the parameters used in the transmission calculation are as follows. The thicknesses of $\mathrm{TiO}_{2}$ and $\mathrm{SiO}_{2}$ are 64 and $103 \mathrm{~nm}$, and their refractive indices are 2.35 and 1.46 , respectively. The initial orientation of the NLC molecules is along the $z$ axis, and the director is rotated in the $y-z$ plane by applying voltage to the NLC defect layer. The thickness of the NLC defect layer is set to $1 \mu \mathrm{m}$. The ordinary and extraordinary refractive indices are $n_{o}=1.53$ and $n_{e}=1.73$, respectively.

\section{RESULTS AND DISCUSSION}

The NLC director distributions in the defect layer were calculated to investigate the electro-optic response of defect mode switching. Figure 2 shows the director distributions of the NLC molecules in the defect layer calculated on the basis of the Franck continuum theory as a function of time. The 


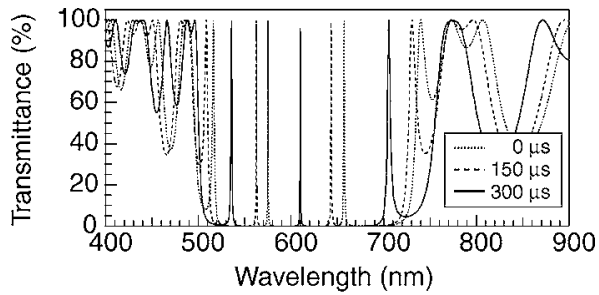

(a)

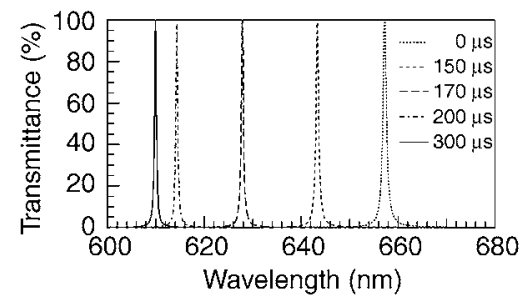

(b)

FIG. 3. Transmission spectra of 1D PC with the NLC defect layer for the applied voltage of $15 \mathrm{~V}$ as a function of time. (b) Detailed view of (a).

reorientations of the NLC molecules for an applied voltage of $15 \mathrm{~V}$ are shown in Fig. 2(a). The initial orientation is along the $z$ axis with a pretilt angle of $0.1^{\circ}$. By applying a voltage of $15 \mathrm{~V}$, the NLC director distributions change in an arched line, and the director angle $\varphi$ increases with the elapsed time. Since the strong anchoring is assumed in the boundary condition, the director angles at both interfaces are fixed. In the reorientation with the applied voltage, the director changes markedly between 120 and $200 \mu$ s. This occurs because the reorientation with applied voltage is based on the dielectric torque being maximum at $\varphi=45^{\circ}$. After applying the voltage of $15 \mathrm{~V}$ for $300 \mu \mathrm{s}$, almost all of the NLC molecules reorient along the $y$ axis, and the molecular directors do not change anymore. Figure 2(b) shows the relaxations of the NLC molecules after removing the applied voltage of $15 \mathrm{~V}$. The relaxations of the NLC molecules start at both ends of the NLC layer, and the director angles gradually return to $0^{\circ}$. Since the relaxation speed decreases with elapsed time, as shown in Fig. 2(b), the reorientation time in the relaxation process is longer than that with the applied voltage. After $5000 \mu \mathrm{s}$, almost all of the NLC molecules are oriented at $0^{\circ}$ along the $z$ axis.

To investigate the transmission properties of the 1D PC with the NLC defect layer, we calculated the transmission spectra using the above director distributions. Figure 3 shows the transmission spectra of the 1D PC with the NLC defect layer for the applied voltage of $15 \mathrm{~V}$ as a function of time. The spectra are calculated from the director distribution shown in Fig. 2(a) and the incident light having a linear polarization along the $z$ axis. This polarization exhibits a large anisotropy in the refractive index when the NLC molecules are reoriented from the $y$ to $z$ axes by applying voltage. In the calculated transmission spectra, the stop band is observed in the spectral range from 520 to $750 \mathrm{~nm}$ in which several defect mode peaks also appear. The full width at half maximum of the defect mode peak is $0.5 \mathrm{~nm}$ and this peak becomes narrower with increasing periodicity of the multilayers. In this calculation, the wavelength resolution $\Delta \lambda$ is set to $0.01 \mathrm{~nm}$. Figure 3(b) is the detailed view of Fig. 3(a)

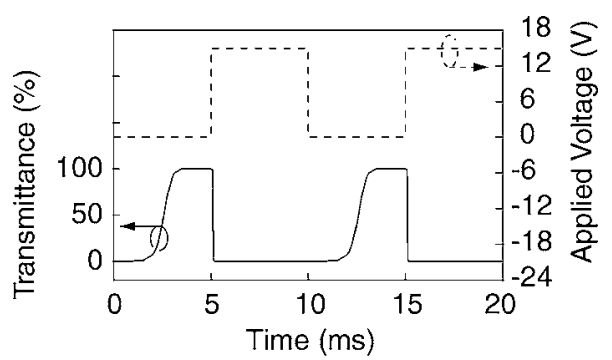

(a)

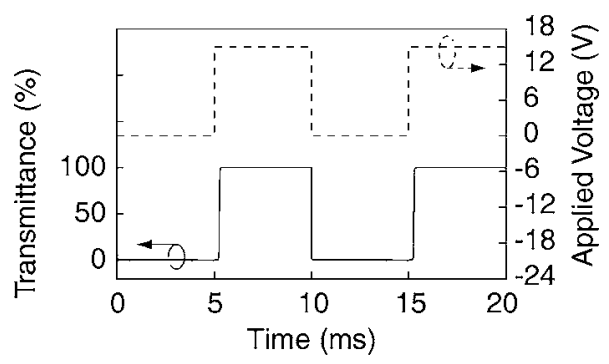

(b)

FIG. 4. Applied voltage wave form and electro-optic response wave form of the defect mode switching in the 1D PC with the NLC defect layer at wavelengths of $657 \mathrm{~nm}$ (a) and $610 \mathrm{~nm}$ (b).

and shows the peak shift on the basis of the molecular reorientation in the NLC defect layer for the applied voltage of $15 \mathrm{~V}$ as a function of time. The defect mode peak shifts to shorter wavelengths with elapsed time. This can be explained by the fact that the refractive index is reduced from the extraordinary to ordinary refractive indices by applying voltage, and the change in refractive index shortens the optical length of the defect layer. In this case, light of $610 \mathrm{~nm}$ wavelength cannot propagate through the 1D PC with the NLC defect layer in the absence of the voltage, but applying voltage allows light propagation through the 1D PC with the NLC defect layer at $610 \mathrm{~nm}$. Therefore, the 1D PC with the NLC defect layer having controllable transmission intensity can be applied in electro-optic switching devices by using the defect mode shift.

Figure 4 shows the electro-optic responses at the defect mode wavelengths of 657 and $610 \mathrm{~nm}$ obtained by applying a square-wave voltage of $15 \mathrm{~V}$ with a frequency of $100 \mathrm{~Hz}$. These results were obtained from the transmittance calculation considering the NLC director reorientations. Figure 4(a) shows the response and applied voltage wave forms at the defect mode wavelength of $657 \mathrm{~nm}$. The incident light of $657 \mathrm{~nm}$ wavelength can propagate in the absence of the applied voltage. By applying voltage, the transmittance at $657 \mathrm{~nm}$ becomes zero, because the defect mode peak quickly shifts to $610 \mathrm{~nm}$, as shown in Fig. 3(b). This shift caused by applying voltage produces the fast decay response shown in Fig. 4(a). After removing the applied voltage, the defect mode peak returns from 610 to $657 \mathrm{~nm}$, and the light of $657 \mathrm{~nm}$ wavelength can propagate through the 1D PC with the NLC defect layer again. However, the rise response based on the relaxation of NLC molecules is very slow. On the other hand, Fig. 4(b) shows the response wave form at the defect mode wavelength of $610 \mathrm{~nm}$. In this case, the 


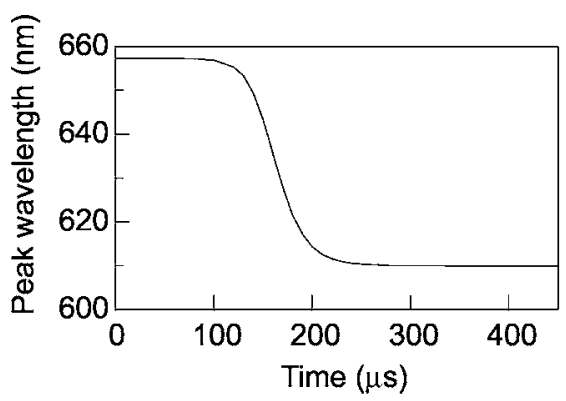

(a)

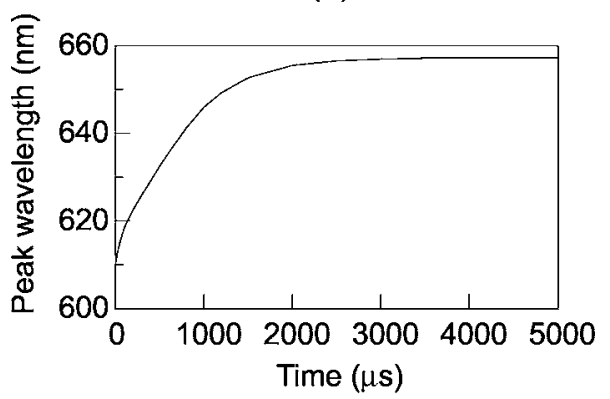

(b)

FIG. 5. Variations of the peak wavelengths with the elapsed time for the applied voltage of $15 \mathrm{~V}$ (a) and after removing the applied voltage (b).

defect mode peak shifts from 657 to $610 \mathrm{~nm}$ with the application of the voltage of $15 \mathrm{~V}$, and the peak returns to $657 \mathrm{~nm}$ after removing the applied voltage. This relation between transmittance and applied voltage is inverted to that at $657 \mathrm{~nm}$. The important point is that both the rise and decay responses at $610 \mathrm{~nm}$ are less than $1 \mathrm{~ms}$ in spite of the use of the same reorientation as that of $657 \mathrm{~nm}$. This is caused by the fact that the peak shift speed is not constant in the molecular reorientations. Figure 5 shows the variation of the peak wavelength with elapsed time. The peak shift due to the applied voltage is $300 \mu \mathrm{s}$, as shown in Fig. 5(a), while the peak shift due to the molecular relaxation requires a longer time than that due to applied voltage, as shown in Fig. 5(b). Note that the shift speed soon after starting is fast in the relaxation process. That is, the switching at $610 \mathrm{~nm}$ is based on the fast director change in the relaxation process after removing applied voltage. Therefore, the fast response of the defect mode switching is realized using the narrow peak shift and the fast part of the relaxation. In contrast, the rise response at $657 \mathrm{~nm}$ is not fast, because the switching at $657 \mathrm{~nm}$ uses the slower part of the relaxation. From the calculations, it was found that the fast response of the defect mode switching was caused by using the fast part of the molecular reorientation.

The applied voltage dependence of the switching response was also calculated to investigate the switching speed in detail. Figure 6 shows the applied voltage wave forms and rise response wave forms of the defect mode switching of the 1D PC with the NLC defect layer at shorter wavelengths as a function of applied voltage. In this case, the defect mode wavelengths for 15,30 , and $45 \mathrm{~V}$ are 610,608 , and $607 \mathrm{~nm}$, respectively, because the director distributions vary slightly different for the applied voltages. In Fig. 6(a), the time delay of the response is observed in the response wave form. Since

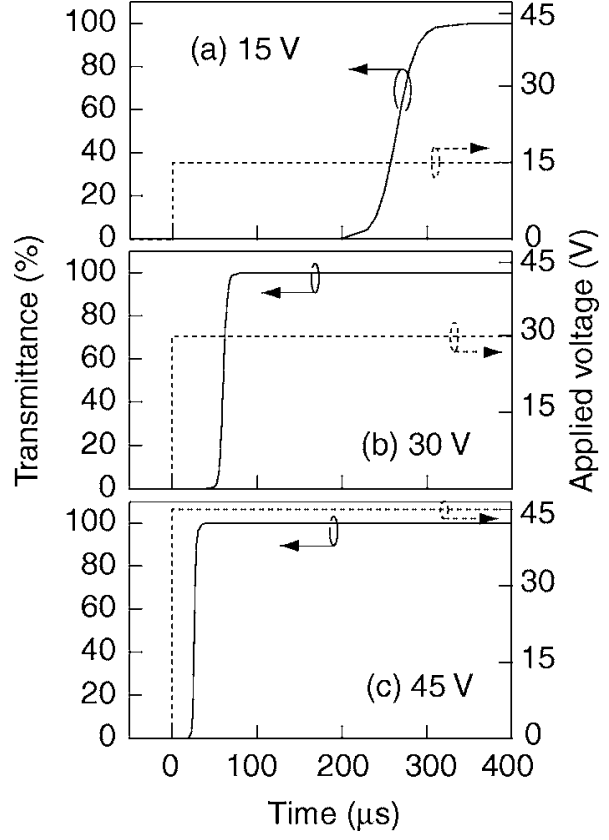

FIG. 6. Rise response wave forms of the defect mode switching of the 1D PC with the NLC defect layer at shorter wavelengths as a function of applied voltage.

the delay time decreases with increasing applied voltage, the delay indicates the speed of the peak shift depending on the applied voltage. Figure 7 shows the decay response wave forms of the defect mode switching of the 1D PC with the NLC defect layer at the shorter wavelengths as a function of applied voltage. The decay responses hardly depend on the applied voltage, because the decay originates from the relaxation process of NLC molecules. Figure 8 shows the voltage dependence of the response time of the defect mode switching at the shorter wavelengths. The response time is defined

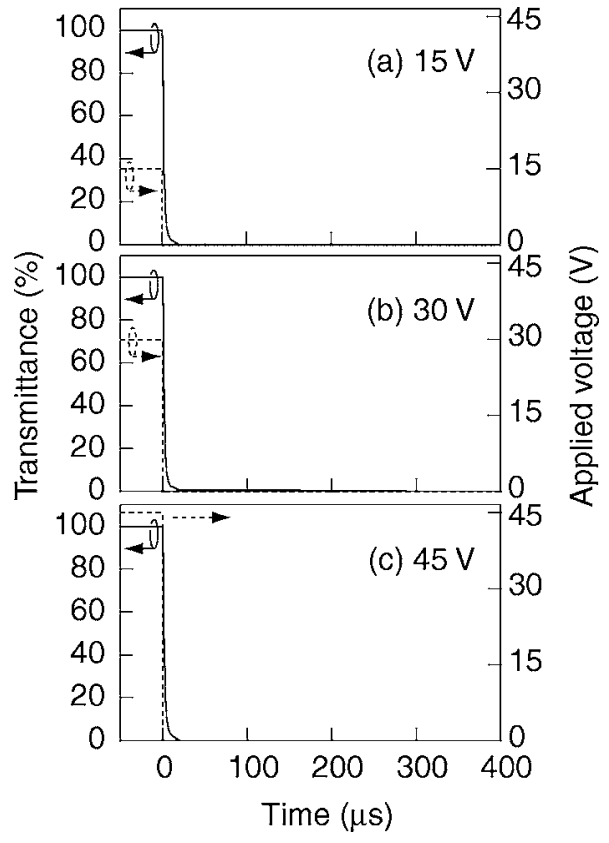

FIG. 7. Decay response wave forms of the defect mode switching of the 1D PC with the NLC defect layer at shorter wavelengths as a function of applied voltage. 


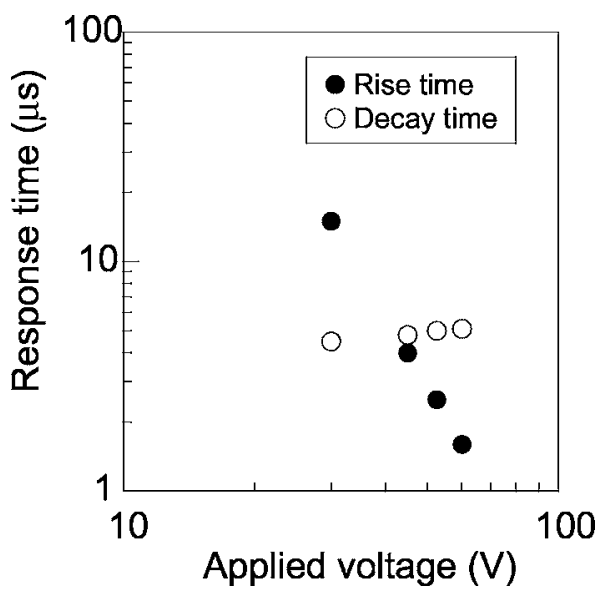

FIG. 8. Voltage dependence of response time of defect mode switching at shorter wavelengths.

as the time required for the change from $10 \%$ to $90 \%$ of total transmission change. Although the rise response is $15 \mu \mathrm{s}$ at $30 \mathrm{~V}$, the rise time is on the order of microseconds at higher voltages. These results show that the rise time decreases with increasing applied voltage. On the other hand, the decay time is approximately $5 \mu$ s and has a weak dependence on the applied voltage. The reason for this is that the reorientation in the absence of applied voltage is based on the NLC molecular relaxation. However, the slight difference in the initial orientation produces a change in the decay response time. The applied voltage dependence of the response time corresponds to the experimental results in our previous report. ${ }^{13}$

Furthermore, we analyzed the viscosity and birefringence dependences of response time to determine the effects of physical parameters on defect mode switching. Figure 9 shows the viscosity dependence of response time at shorter wavelengths for the applied voltage of $45 \mathrm{~V}$. Both the rise and decay times are proportional to viscosity. Since the molecules in the defect layer simply reorient from planar to homeotropic alignment, the viscosity dependence of response time exhibits the same trend as a general cell having a change in orientation from planar to homeotropic. Similarly,

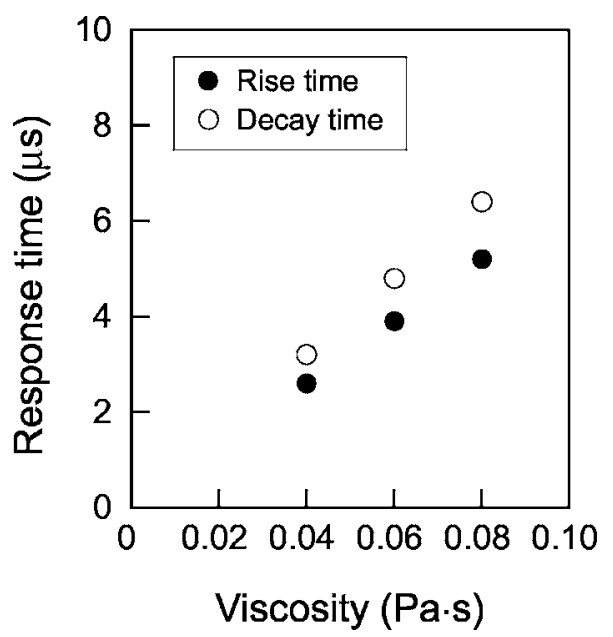

FIG. 9. Dependence of response time of defect mode switching on viscosity of NLC at shorter wavelengths for the applied voltage of $45 \mathrm{~V}$.

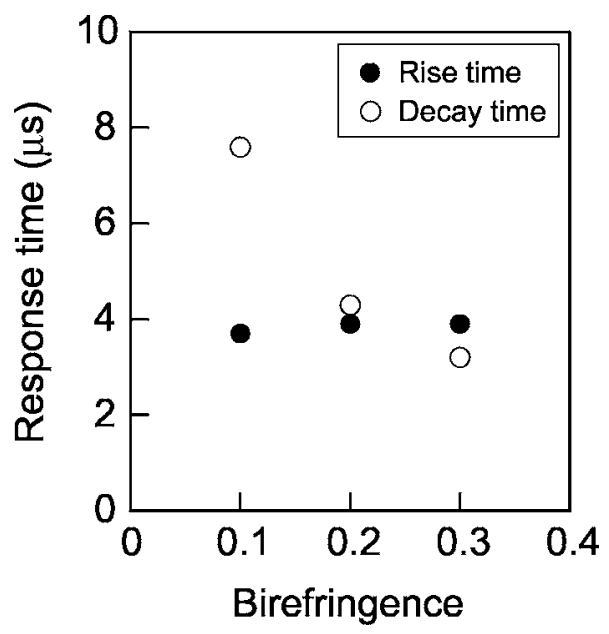

FIG. 10. Birefringence dependence of response time of defect mode switching at shorter wavelengths for the applied voltage of $45 \mathrm{~V}$ and $\gamma$ $=0.06 \mathrm{~Pa} \mathrm{~s}$.

it can be speculated that the response time decreased with increasing elastic constants and dielectric anisotropy. On the other hand, the birefringence affects response time, unlike in the case of a general LC switching, because the defect mode switching is based on the peak shift induced by the change in refractive index. That is, it is considered that the increase in the birefringence with the same director distribution produces a larger peak shift and a faster response. Figure 10 shows the birefringence dependence of the response time of defect mode switching at the shorter wavelengths for the applied voltage of $45 \mathrm{~V}$ and $\gamma=0.06 \mathrm{~Pa}$ s. The rise times are approximately the same, whereas the decay time decreases with increasing birefringence. The decay response is based on the maximum rate of change of the peak shift at the starting point of reorientation, as shown in Fig. 5(b); thus, the increase in the birefringence has a strong effect on the decay time. In contrast, the rise response is based on the minimum rate of change of the peak shift near the director angle of $90^{\circ}$, for example, in the vicinity of $250 \mu$ s in Fig. 5(a). That is, since the rise response always uses the end of the peak shift, the rate of change of the peak shift shows a certain value independent of birefringence. From these results, the optical properties of the defect mode switching of the 1D PC with the NLC defect layer were clarified by the dynamic analysis that takes into account the NLC director distributions.

\section{CONCLUSIONS}

The defect mode switching of a 1D PC with a NLC defect layer was analyzed theoretically. The transmission characteristics were calculated by considering the NLC director distributions. Since the molecular reorientation in the NLC defect layer results in a change in the optical length, the defect mode peaks are shifted by applying voltage. Using the peak shift and the fast part of the relaxation, the defect mode switching showed a response on the order of the microseconds. The fast response was observed not only in the rise wave form but also in the decay wave form. These results corresponded to the experimental results in our previous report. Furthermore, the theoretical analyses enabled us to 
clarify the dependences of the switching response on the several physical parameters. This analysis procedure is potentially a useful method for the investigation of PCs with a LC defect layer.

${ }^{1}$ E. Yablonovitch, Phys. Rev. Lett. 58, 2059 (1987).

${ }^{2}$ S. John, Phys. Rev. Lett. 58, 2486 (1987).

${ }^{3}$ S. Noda, K. Tomoda, N. Yamamoto, and A. Chutinan, Science 289, 604 (2000).

${ }^{4}$ J. S. Foresi et al., Nature (London) 390, 143 (1997).

${ }^{5}$ J. P. Dowling, M. Scalora, M. J. Bloemer, and C. M. Bowden, J. Appl. Phys. 75, 1896 (1994).

${ }^{6}$ K. Yoshino, Y. Shimoda, Y. Kawagishi, K. Nakayama, and M. Ozaki, Appl. Phys. Lett. 75, 932 (1999).
${ }^{7}$ K. Yoshino, S. Satoh, Y. Shimoda, Y. Kawagishi, K. Nakayama, and M. Ozaki, Jpn. J. Appl. Phys., Part 2 38, L961 (1999).

${ }^{8}$ K. Busch and S. John, Phys. Rev. Lett. 83, 967 (1999).

${ }^{9}$ Y. Shimoda, M. Ozaki, and K. Yoshino, Appl. Phys. Lett. 79, 3627 (2001).

${ }^{10}$ M. Ozaki, Y. Shimoda, M. Kasano, and K. Yoshino, Adv. Mater. (Weinheim, Ger.) 14, 514 (2002).

${ }^{11}$ R. Ozaki, T. Matsui, M. Ozaki, and K. Yoshino, Jpn. J. Appl. Phys., Part 2 41, L1482 (2002).

${ }^{12}$ R. Ozaki, T. Matsui, M. Ozaki, and K. Yoshino, Appl. Phys. Lett. 82, 3593 (2003).

${ }^{13}$ R. Ozaki, M. Ozaki, and K. Yoshino, Jpn. J. Appl. Phys., Part 2 42, L669 (2003).

${ }^{14}$ F. C. Frank, Discuss. Faraday Soc. 25, 19 (1958).

${ }^{15}$ D. W. Berreman, J. Opt. Soc. Am. 63, 1374 (1973). 